\title{
Para repensar la profundización democrática: ideales, conceptos y desafíos analíticos Alejandro Monsiváis Carrillo*
}

\section{Resumen}

Este trabajo tiene como propósito discutir los desafíos conceptuales que enfrenta una teoría de la profundización democrática. En primera instancia se discuten las contribuciones y los límites tanto normativos como analíticos de la noción de "calidad de la democracia”. Enseguida se argumenta que es necesario reconstruir los preceptos asociados con el ideal de la democracia para discernir los ideales regulativos que pueden ser objeto específico de escrutinio. La apuesta consiste en abrir nuevas agendas de investigación en torno a los conceptos que son parte del ideal de una democracia vital y robustecida. Esto implica repensar al menos tres elementos constitutivos del gobierno democrático: la participación, la competencia y el control popular de la acción pública.

Palabras clave: calidad de la democracia, regímenes políticos, teoría normativa, análisis político, análisis conceptual.

\begin{abstract}
The purpose of this paper is to discuss the conceptual frontlines of a theory of the deepening of democracy. First, the contributions and limitations of the literature on the quality of democracy are discussed. Then, it is argued the necessity of reconstructing the principles related to the idea of democracy in order to discern the regulative ideals that should be subjects of empirical scrutiny. The challenge is to open new research agendas centered on those elements that constitute a lively and robust democracy. This will imply the task of rethinking the concepts of participation, political competition, and popular control of public action.
\end{abstract}

Keywords: quality of democracy, political regimes, normative theory, political analysis, conceptual analysis.

* Profesor-investigador de El Colegio de la Frontera Norte. Correo electrónico: amonsi@colef.mx 


\section{Introducción}

$\mathrm{Al}$ menos dos formas de teorización convergen en el análisis político. Una de ellas es la que proviene del escrutinio de observaciones empíricas; la otra se origina en la reflexión en torno al significado, coherencia y relevancia de los ideales y los preceptos normativos de la vida política (Rawls, 1999, Dryzek, Honig y Phillips, 2006). Estas dos formas de razonamiento se conducen, habitualmente, por rutas separadas. El interés que ha suscitado y la amplia difusión que ha tenido el concepto de calidad de la democracia durante la última década se deben, en buena medida, a que se trata de un concepto que tiene fuertes conexiones, simultáneamente, con la teoría política normativa y con el análisis político empírico.

Cuando se habla de la "calidad" de los regímenes democráticos se hace alusión a las pautas de desempeño y funcionamiento que son inferidas a partir de distintos tipos de evidencia; al mismo tiempo, se pone en juego un estándar de calidad que cualifica a lo que se observa en función de lo que podría o debería de ser. Este doble arraigo de la noción de la "calidad de la democracia” ha dado lugar al surgimiento y la exploración de nuevas agendas en el estudio de los regímenes políticos. Sin embargo, varias dificultades y algunos desafíos que enfrenta la noción de la "calidad de la democracia" se originan, fundamentalmente, en la tensión que surge entre su anclaje teórico-normativo y su propósito de servir para el análisis político.

$\mathrm{Al}$ esforzarse por elaborar un modelo integrado y comprensivo de una democracia de "calidad", los contornos analíticos de esta noción han quedado difusos y la teoría ha encontrado dificultades para pasar de la descripción y la clasificación a la especificación de explicaciones causales (Munck, 2004). Esto no significa que el debate esté agotado ni que resulte ocioso continuar indagando en lo que representa conceptualizar y explicar la "democraticidad" del régimen. En este trabajo, mi propósito es insistir en que el estudio de la calidad de la democracia todavía tiene agendas de investigación abiertas que desarrollar. Mi argumento será que, ante todo, es necesario reconstruir los preceptos asociados con el ideal de la democracia para discernir, así, los ideales regulativos que pueden ser objeto específico de escrutinio. La apuesta consiste en abrir nuevas agendas de investigación en torno a los conceptos que son parte del ideal de una democracia vital y robustecida. 


\section{Sobre la "calidad" de la democracia: alcances y límites de una noción}

Un régimen que emerge de una fase de autoritarismo, en el que se constata que la democracia se convierte en the only game in town (Linz y Stepan, 1996), y en el que se realizan con regularidad elecciones competitivas, no es un régimen en el que, por necesidad, las condiciones de vida de la población vayan a mejorar, en el que la corrupción y la impunidad se erradicarán por decreto, o en el que los gobernantes se vayan a convertir en políticos visionarios y responsivos. Las últimas dos décadas han mostrado, lo mismo en América Latina que en otras democracias de la "tercera ola", que un régimen requiere algo más que elecciones libres y competitivas para producir resultados públicamente significativos.

Constatar que un régimen puede transitar del autoritarismo a la democracia sin que eso implique que se alcanzan a cumplir las expectativas asociadas al cambio político, genera múltiples interrogantes: ¿qué requiere una democracia, además de elecciones libres, regulares y competitivas, para dar los resultados que se esperan de ella? ¿No se supone que una democracia es un régimen idóneo para promover el interés público y el beneficio de las mayorías? ¿Es compatible la democracia con la desigualdad y la pobreza, con la corrupción y la arbitrariedad en el ejercicio del gobierno, o con la vulnerabilidad de los derechos humanos? ¿Hasta qué punto? ¿Por qué razón? A raíz de preguntas como éstas ha sido necesario repensar las articulaciones entre el régimen político, las elecciones competitivas, el Estado de derecho y las políticas públicas.

El desempeño de las democracias emergentes contribuyó a revitalizar el interés por la conceptualización de los regímenes políticos: ¿̇ómo clasificar a estos regímenes que tienen los atributos de la poliarquía pero que conviven con la desigualdad, la ineficacia gubernamental y la inestabilidad política? Los regímenes en cuestión han sido etiquetados como "democracias liberales" (Zakaria, 2003), "democracias electorales" (Diamond, 1999) y "democracias deficientes" (Croissant y Merkel, 2004), entre otras variedades. Estas etiquetas designan, con mayor o menor sistematicidad, a los atributos que podían distinguir a las "democracias con adjetivos" -los subtipos disminuidos del régimen democrático- de las democracias propiamente dichas (Collier y Levitsky, 1997). 
La revitalización que ha tenido el análisis conceptual de los regímenes políticos y sus atributos ha dado lugar a contribuciones significativas. Una de las aportaciones conceptuales más originales ha sido el concepto de autoritarismo competitivo o electoral (Levistky y Way, 2010; Schedler, 2006). Hasta hace poco parecía que las elecciones regulares y competitivas eran atributos exclusivos de las poliarquías; el análisis comparado de países del continente africano, el Medio Oriente, la ex Unión Soviética, Europa del Este y América Latina muestra que los dictadores y las élites autócratas también pueden recurrir a las elecciones para sostener su poder y reproducir su dominio (Levitsky y Way, 2010) — hasta hace poco más de una década, más que una “dictadura perfecta”, México podía considerarse un caso singular y bien logrado de "autoritarismo competitivo".

Uno de los conceptos más importantes, en este contexto, es el de calidad de la democracia. Esta noción ha desencadenado productivos debates y alentado nuevas agendas de investigación. Esto se debe, en primer lugar, a que la tarea de identificar los preceptos regulativos de una comprensión integral y renovada de una democracia ha promovido el diálogo entre la teoría política normativa y el análisis comparado. El ímpetu por clarificar las bases normativas de la calidad de la democracia introdujo en la reflexión sobre los regímenes políticos preceptos como los de desarrollo humano, derechos humanos, legalidad y justicia (Maravall y Przeworski, 2003; Méndez, Pinheiro y O’Donnell, 1999; O’Donnell, Vargas-Cullel e Iazzetta, 2004; PNUD, 2004). En segundo lugar, el concepto de calidad de la democracia ha sido de utilidad para integrar bajo un mismo marco de referencia temáticas emergentes e innovadoras: la fortaleza del Estado, la administración y procuración de justica, la transparencia y el acceso a la información, y el funcionamiento de las distintas modalidades de rendición de cuentas (Bergman y Whitehead, 2009; Mainwaring y Welna, 2003).

Con todo, el desarrollo de una agenda de investigación centrada específicamente en la calidad de la democracia ha tenido dificultades para sobrepasar algunos obstáculos clave. Uno de ellos, en primera instancia, está ligado a la teorización causal. De acuerdo con Munk (2004), el estudio de la calidad de la democracia ha adoptado un enfoque prioritariamente descriptivo y clasificatorio. Esto es comprensible en la medida en que los trabajos iniciales comenzaron estableciendo la agenda de investigación 
y aportando elementos para el estudio empírico de esta cuestión (PNUD, 2004; O’Donnell, Vargas-Cullel e Iazzeta, 2004). En paralelo, la multidimensionalidad propia de este fenómeno ha dado lugar al desarrollo de diversos modelos de evaluación de la "calidad" de la democracia. Es el caso de los estudios promovidos por el Instituto Internacional para la Democracia y la Asistencia Electoral, que emplean un esquema formulado por Landmann (2009) en el que se desglosa un marco de trabajo integrado por los principios de la democracia, valores mediadores, cuatro pilares de un régimen y hasta 90 preguntas específicas.

El énfasis en la evaluación de la calidad de la democracia ha estado acompañado, de igual forma, por diversos intentos de formular explicaciones causales. Mainwaring y Perez-Liñán (2008), por ejemplo, señalan que el nivel de democracia alcanzado por los países latinoamericanos entre 1978 y 2004 se puede explicar a partir de los legados de cada caso durante la "primera" (1900-1944) y la "segunda" (1945-1977) de la democracia. De igual manera, destaca la teorización desarrollada por Morlino (2011) en la que se formulan diversas hipótesis acerca de los procesos de transición, consolidación y fortalecimiento de la calidad de la democracia. Además de esfuerzos integradores como los de Morlino, y diversas aportaciones puntuales, todavía es necesario formular un sistema hipotético que explique la variabilidad en el fenómeno de interés. Esto se debe, en gran medida, a que el objeto de estudio ha quedado definido de manera imprecisa. Es decir, existen dificultades para saber a ciencia cierta qué es lo que se tendría que explicar si se quisiera encontrar los determinantes o las causas de la calidad de la democracia.

Una segunda dificultad asociada a la "calidad de la democracia" es, de hecho, que se trata de una noción que no ha sido objeto de una especificación conceptual rigurosa. En un plano coloquial, una democracia de calidad es simplemente un régimen en el que las dinámicas de la vida pública se acercan a lo que intuitivamente se asume o se piensa como deseable. Al tratar de llegar a una formulación sistemática, tiende a dibujarse una noción integrada por atributos del régimen político, del Estado de derecho y del desempeño gubernamental. Esto contribuye a crear problemas de diferenciación analítica —o, para emplear la expresión de Sartori (1970), de "estiramiento conceptual"- . "Estirar" el concepto de "calidad de la democracia” supone hacerle abarcar componentes que podrían ser propios 
de otros conceptos. Las dos contribuciones teóricas más importantes al estudio de la calidad democrática adolecen de este problema. Por un lado, O’Donnell (2004) colapsa en el estudio de la calidad de la democracia cuestiones vinculadas con el Estado, la burocracia y el régimen democrático. Por otro, en una aproximación por demás interesante, Morlino (2005, 2011) inserta dentro de la noción de calidad de la democracia aspectos como la legalidad y la rendición de cuentas, la responsividad de los gobernantes y los resultados sustantivos de las políticas públicas. En pocas palabras, la conceptualización que se ha hecho de esta noción tiene como desventaja que colapsa en una misma definición aquellas relaciones empíricas que deberían ser diferenciadas conceptual y analíticamente (Munck, 2007; Mazzuca, 2007).

En tercer lugar, estrechamente vinculado con el proceso de especificación conceptual, se encuentra la cuestión de las reglas de agregación de indicadores. Cuando se está operacionalizando un concepto complejo, luego de desagregar horizontalmente las dimensiones que componen ese concepto, es preciso desprender de cada una de esas dimensiones variables e indicadores empíricos. La tarea de agregación consiste en seguir la ruta inversa, desde los indicadores empíricos hasta el concepto básico. Las reglas de agregación que se utilicen para ascender desde los indicadores empíricos hasta la medida integrada del concepto deben responder a criterios teóricos (Munck y Verkuilen, 2002).

En el estudio de la calidad de la democracia, diversos trabajos siguen un procedimiento estándar: una vez que se ha reunido una amplia variedad de indicadores, se emplean diversas reglas hasta producir una medida única de la calidad de la democracia. El problema no es simplemente de cómputo. Los analistas son cada vez más acuciosos en el proceso de construcción de índices compuestos (Levine y Molina, 2007; Mainwaring, Scully y VargasCullel, 2010). La cuestión radica, como señala Munck, en saber si "acaso es deseable reducir múltiples medidas en una sola” (2006:36). Lo que está implícito es el supuesto de que el concepto de calidad de la democracia, aunque esté integrado por múltiples dimensiones, en realidad constituye un solo vector en torno al cual convergen todos los indicadores. La dificultad estriba en que, intuitivamente, se puede pensar que el concepto de calidad de la democracia es multidimensional; esto implica que no debería esperarse que una sola medida pudiera describir a la calidad de la democracia. 
Sirva de ilustración el Índice de Desarrollo Democrático de América Latina. Éste es un índice compuesto que proporciona una medida única del nivel de desarrollo democrático para 18 países latinoamericanos, que elabora la Fundación Konrad Adenauer y la empresa de consultoría PoliLat. Este índice se compone de cuatro dimensiones: a) la legalidad del régimen democrático; $b$ ) respeto de los derechos políticos y libertades civiles; $c$ ) calidad institucional y el grado de eficiencia política; y $d$ ) el ejercicio de poder efectivo para gobernar. Esta última dimensión a su vez se divide en dos subdimensiones: capacidad para generar políticas que aseguren bienestar y capacidad para generar políticas que aseguren eficiencia económica.

Son diversos los méritos que tiene este índice: por un lado, ha sistematizado una amplia variedad de datos institucionales, de opinión pública y socioeconómicos en una medida estandarizada, que permiten comparar a 18 países de la región en una serie temporal ininterrumpida desde 2002. A este índice se le puede criticar, sin embargo, que proporciona una comprensión de la democracia tal, en la que se asume que una amplia variedad de datos pueden ser agregados en torno a una sola dimensión. Si el desarrollo democrático es multidimensional, como este índice lo señala explícitamente, puede resultar una pérdida de información el que esa multidimensionalidad quede reducida a un solo vector de variabilidad. La construcción de un índice no es un problema meramente técnico; tiene que estar basada en una estructura conceptual debidamente definida.

Las tres dificultades que hemos señalado constituyen problemas de fondo para una teoría sobre la calidad de la democracia. Por un lado, la especificación conceptual ha sido imprecisa y plausiblemente requiere una diferenciación analítica más cuidadosa. Por otro, la construcción de indicadores no podrá generar medidas certeras y eficientes si la especificación conceptual es imprecisa. Finalmente, sin un concepto con una estructura definida de manera convincente, el desarrollo de una teoría explicativa y la puesta a prueba de hipótesis empíricas se verán limitados.

En estas circunstancias, hablar de la "calidad" de la democracia corre el riesgo de convertirse en una expresión coloquial, despojada de penetración analítica. Si esto sucede, el precio que se ha de que pagar será alto, pues las discusiones sobre la "calidad" no carecen de fundamento. La literatura sobre la calidad de la democracia se origina en una inquietud de orden normativo: es necesario exigir más a la democracia, demandarle que 
esté a la altura de las expectativas que ha generado. La dificultad radica en que la discusión académica no ha conseguido resolver satisfactoriamente los problemas de teorización a los que se ha enfrentado.

El principal desafío que enfrenta la noción de la calidad de la democracia es el de definir criterios sistemáticos de evaluación. Este reto parecería ser fundamentalmente de orden conceptual: hace falta delimitar mejor los contornos de una democracia de calidad, identificar con mayor detenimiento sus componentes y afinar sus indicadores empíricos. La tarea es, sin embargo, más complicada. La democracia es un concepto controvertido, esencialmente disputable (Collier, Hidalgo y Maciuceanu, 2006). Especificar los contenidos de la calidad de la democracia supone adentrarse en una exploración teórico-normativa. Implica hacer distinciones analíticas y reconstruir el sentido de los ideales democráticos.

\section{Repensando la profundización democrática}

Uno de los principales desafíos para la investigación en torno a la calidad de la democracia es ofrecer respuestas a una pregunta clave: ¿qué forma adopta una democracia vital y vibrante? Una pregunta de esta índole desemboca en un campo de múltiples interrogantes. Los contenidos y los sentidos de la democracia son controversiales. Sin embargo, ofrecer respuestas a esta pregunta es vital para formular planteamientos heurísticos que orienten la investigación empírica.

El concepto de régimen democrático constituye el anclaje analítico necesario para profundizar en la evaluación de la calidad de la democracia. Este es el punto de partida para reconstruir los componentes normativos de una democracia vibrante y robusta. De acuerdo con el planteamiento de Mainwaring, Brinks y Pérez-Liñan (2007), la democracia es un régimen que está compuesto por cuatro condiciones necesarias y suficientes: hay elecciones libres y competitivas; existen garantías para la participación inclusiva de los adultos; se protegen las libertades civiles y políticas; y las autoridades gobiernan sin interferencias de agentes no electos - por ejemplo, los grupos de interés- - Si alguna de estas condiciones no está presente, el régimen es un régimen autoritario; si alguna de esas condiciones se presenta de manera parcial, se trata de un sistema semidemocrático. 
El concepto de régimen democrático funge como un instrumento de clasificación: proporciona criterios que permiten distinguir entre regímenes políticos. La calidad de la democracia, entonces, sólo puede ser evaluada en los regímenes que cumplen con las características mínimas de la democracia. Cabe advertir que existen medidas numéricas del grado de democracia de un sistema político, con las que se pueden hacer distinciones más finas entre los regímenes: Freedom House, Polity Iv, etcétera.; pero estas medidas no fueron diseñadas con el propósito explícito de evaluar la calidad de las democracias. Sigue pendiente, por lo tanto, una conceptualización que proporcione mayor capacidad de diferenciación analítica. Concretamente, el desafío de una teoría de la calidad de la democracia reside en analizar la democraticidad de las instituciones y los procesos políticos que acontecen en el marco de ese régimen.

Para proseguir con la discusión es preciso, antes, una precisión analítica: aunque las democracias constitucionales contemporáneas sean el producto de la articulación entre el Estado de derecho y el principio de la soberanía popular (Habermas, 1998; Ferejohn y Pasquino, 2003), es preciso distinguirlos claramente. Para proceder con cierta celeridad, digamos que la democracia es un principio para organizar el ejercicio del gobierno y la autoridad pública, mientras que el Estado es la entidad que tiene soberanía sobre un espacio geográfico claramente delimitado. Si se habla de un "Estado de derecho", entonces cabe añadir que, conforme a los preceptos de justicia contemporáneos, el Estado ejerce un poder delimitado y circunscrito por una Constitución, en aras de proteger la libertad y los derechos de los individuos. Esto implica que la burocracia estatal debe estar profesionalizada y conducirse con certeza jurídica; y que debe estar sometida a mecanismos de control y vigilancia que aseguren que la gestión pública se conduce con apego a los principios de eficacia, eficiencia, legalidad, transparencia y equidad.

Una vez establecida la diferencia entre el régimen democrático y el Estado de derecho, ¿cómo entender, entonces, el carácter democrático de los procesos políticos? De manera congruente con las bases normativas del principio democrático, es necesario partir de la igualdad política (Christiano, 1996; Dahl, 1971; Held, 2006; acerca de las implicaciones y consecuencias de este punto de partida, véase Przeworski, 2010). Compleja y difícil de asir, la noción de igualdad política es un parámetro regulativo. 
Esta noción remite a la idea de que, con independencia de la prosperidad y el bienestar del que goce cada persona en su vida privada, la vida pública habrá de estar conducida de tal forma que existan condiciones y oportunidades para que cada persona intervenga, en condiciones de equidad, en la configuración de las leyes y políticas públicas. Por lo tanto, la vitalidad y densidad democrática de un régimen político dependen del grado en que las decisiones colectivas, las leyes y las políticas públicas se adopten, formulen e implementen bajo condiciones de equidad política efectiva.

La finalidad de la política democrática es producir decisiones colectivas legítimas, susceptibles de ser objeto de escrutinio y la rendición de cuentas pública, a partir de condiciones de equidad política. El énfasis aquí está puesto en las condiciones y características del proceso de participación, decisión y evaluación políticas. Por ende, la evaluación de la calidad de la democracia debe recaer sobre los procedimientos, las condiciones y los atributos de los procesos políticos. La democracia genera decisiones colectivas en el sentido de que determina cuáles alternativas resultan ganadoras y cuáles perdedoras. En sentido estricto, lo relevante no es su contenido, sino las condiciones en las que fueron adoptadas. Las leyes y políticas pueden ser certeras y precisas, o bien, veleidosas y fútiles. Sin embargo, si el procedimiento es correcto, la decisión es legítima y el resultado obligatorio (Christiano, 2004).

La tesis de fondo es que es la calidad de los procesos de formación de la agenda pública, deliberación y decisión lo que importa en la política democrática. Esta tesis es controvertida, pero considerablemente certera. Se podría objetar a este planteamiento que conduce a una concepción procedimental de la democracia y, de esta manera, a un modelo estrecho y reduccionista de la política democrática. Sin embargo, el carácter procedimental de la democracia no implica dotar al régimen de una connotación elitista, no-participativa o meramente agregativa. La concepción procedimental de la democracia es compatible con la participación popular, los mecanismos de democracia directa y la deliberación pública. El punto es que los resultados de los procesos democráticos no son una condición necesaria ni suficiente del concepto de democracia. Los resultados del procedimiento democrático deben evaluarse aparte.

Esta tesis se contradice con otra concepción, en la que se equipara a la democracia con un régimen que progresivamente produce condiciones 
reales de igualdad socioeconómica. Si la democracia es un régimen de individuos que se reconocen como libres e iguales, desde este punto de vista el soporte necesario de esa igualdad tendría que ser de tipo social y económico. La democracia, en efecto, tendría que ser capaz de dar lugar a gobiernos que tomen mejores decisiones; esto es, que sean capaces de responder de manera efectiva a las demandas del público y esto, en general, implica mejorar las condiciones de vida de la población. La capacidad de la democracia para responder a las demandas sociales parece ser clave para su sustentabilidad en el mediano y largo plazos (PNUD, 2010). Sin embargo, la relación entre el régimen y los resultados de las políticas públicas es, ante todo, un objeto de investigación empírica.

Una comprensión sustantiva de la democracia, ligada al modelo clásico de la idea del autogobierno, también se manifestaría en contra de evaluar la calidad de la democracia a partir de las condiciones y los procedimientos de elección colectiva. Esta comprensión se origina en la metáfora de la voluntad general y afirma que la calidad de la democracia debería observarse no en el proceso de agregar las preferencias individuales, sino en el consenso emanado de la expresión política de una voluntad colectiva. Esta concepción es inconsistente en múltiples sentidos -la concepción elitista de Schumpeter, por ejemplo, se sustenta sobre una de las críticas más mordaces a esta concepción de la democracia (Schumpeter, 1971)-. Por principio de cuentas, dicha concepción es incompatible con una teoría de la democracia apropiada para sociedades plurales, sociocultural e ideológicamente diversas (Young, 2000). Por lo tanto, es necesario desligar a la democracia de las connotaciones consensuales y de homogeneidad moral e ideológica del colectivo asociadas a la idea de la voluntad general.

El problema de fondo con una concepción de la democracia entendida como la manifestación de una voluntad colectiva radica en la tensión que hay entre la racionalidad de las preferencias individuales y la racionalidad de una decisión colectiva. La teoría de la elección social ha demostrado que ningún procedimiento de agregación que pretenda cumplir con un conjunto determinado de condiciones de imparcialidad garantiza una traducción inequívoca entre la distribución de preferencias individuales y el resultado de una decisión colectiva (Austen-Smith y Banks, 2000). Este planteamiento ha servido para generar suspicacias acerca del contenido "populista” de la democracia (Riker, 1982). En la práctica, múltiples arre- 
glos institucionales contribuyen a evitar las consecuencias incoherentes de todo procedimiento de decisión. En un sentido estrictamente normativo, esta discusión contribuye a poner énfasis en las condiciones y dinámicas del proceso político, desligando a la evaluación intrínseca del procedimiento democrático del contenido de las decisiones colectivas.

Por otra parte, es indispensable darle centralidad a la calidad del proceso político si se quiere explicar el mecanismo endógeno que puede hacer sostenible al régimen democrático. En este punto sigo el argumento de Przeworski (1991): la democracia es consecuencia de un equilibrio estratégico; no se puede imponer desde fuera a una comunidad política. Debe afianzarse internamente como un mecanismo de coordinación colectiva y autorización política. La democracia es frágil e inestable, pues depende de que todos los actores implicados —en especial aquellos que detentan ciertos intereses y tienen el poder y el dinero para hacerlos valer - renuncien a imponer unilateralmente su voluntad. Esto implica que la democracia debe constituir la estrategia dominante para los poderosos, incluso si se depende exclusivamente del cálculo egoísta y utilitario de cada individuo. Por este motivo, los costos de perseguir los propios fines por fuera del régimen deben ser considerablemente mayores a los que implica asumir una derrota temporal de las alternativas preferidas. Este efecto se consigue si las condiciones de elección y decisión colectivas están adecuadamente niveladas, son imparciales y dejan abierta la posibilidad en un futuro cercano de revertir el resultado. Un sistema regular, imparcial y competitivo de procesos de elección colectiva puede generar incentivos para que los agentes políticos inviertan en hacer prevalecer sus opciones preferidas en el marco de la democracia.

Una democracia de calidad es, entonces, un régimen en el que los debates públicos, la formulación de las leyes, las decisiones colectivas y la elección de las políticas públicas se realizan con base en condiciones robustecidas de equidad e igualdad políticas. Las implicaciones de este planteamiento se discuten en las dos secciones siguientes.

\section{Los componentes de la vitalidad democrática}

Para identificar los componentes de la vitalidad democrática es necesario discernir qué elementos de los procesos políticos que vayan más allá de la 
realización de elecciones libres y competitivas pueden ser consistentes con los principios democráticos. Este es el procedimiento que ha empleado Munck (2011) para conceptualizar nuevos estándares de la calidad de la democracia. En este caso, si lo que dota de fortaleza a la democracia es la vitalidad con la que se concreten y manifiesten condiciones de equidad e igualdad políticas en los procesos públicos, es necesario identificar cuáles son esas condiciones que pueden observarse con un grado variable de intensidad. En esta parte señalaré que existen tres componentes de los procesos de acceso y ejercicio del gobierno y la autoridad públicos que deben ser considerados como arenas de la profundización democrática: participación, competencia y control popular de la acción pública.

Los primeros dos elementos, participación y competencia, provienen del concepto de poliarquía de Robert Dahl (1971, 1989). En gran medida estos dos componentes de la democracia condensan y capturan las condiciones necesarias para que las decisiones públicas y colectivas, de carácter vinculante, sean congruentes con los ideales de autogobierno y autonomía política. La tesis de Dahl continúa teniendo vigencia: la democracia es un sistema en el cual los individuos deben poder constituir de manera libre sus preferencias políticas, sopesarlas en el proceso político y hacer que esas preferencias determinen los cursos de acción que habrá de seguir la colectividad. Esto supone disponer de fuentes alternativas de información, libertades de asociación y comunicación, que los principales cargos de gobierno sean electos, que el sufragio sea universal, y que haya elecciones libres, regulares y competitivas. Estas son las condiciones elementales para la construcción de una esfera pública vibrante.

La participación política es clave para robustecer a la democracia. La libertad para intervenir en los asuntos públicos es un derecho fundamental, que se puede ejercer de manera tanto positiva como negativa —el derecho a abstenerse es tan legítimo como el derecho a participar-. Ceteris paribus, se presume que la política democrática se beneficia del involucramiento activo, constante y diversificado de los ciudadanos. La expresión primordial del derecho a la participación es el acto de votar y ser votado/a. El estudio de la participación electoral da cuenta de la legitimidad del régimen entre la población, pero también de la manera en que los ciudadanos se han apropiado efectivamente de sus derechos políticos. Como indica Coppedge (2007), un indicador robusto de la democracia debe- 
ría incorporar indicadores no solamente acerca de la universalidad del sufragio, sino también de la generalización de las capacidades efectivas para ejercerlo y tener influencia política. El estudio de la manipulación electoral o la compra y coacción del voto, consecuentemente, tendría que figurar entre los tópicos ligados a la calidad de la democracia.

Desde luego, la participación democráticamente significativa no está limitada a la participación electoral. Los sistemas políticos contemporáneos están compuestos por múltiples foros públicos, en los que se consulta, se decide y se actúa. Por lo tanto, el estudio integral de la participación política necesita incorporar a la evaluación del régimen democrático las distintas variedades de instrumentos participativos que han emergido en las últimas décadas. En este plano entran las formas de participación públicopolítica que van desde la intervención en las asambleas barriales hasta las movilizaciones generalizadas de protesta o las formas de comunicación y coordinación con fines estrictamente políticos que se facilitan por el uso de la tecnología. En pocas palabras, es necesario conocer cómo surgen, cómo funcionan y qué consecuencias tienen los instrumentos, espacios y foros públicos en términos de promover la participación y la colaboración en la definición e implementación de políticas (Fung, 2006; Smith, 2009).

Los principios de oposición y competencia aparecen en este punto. La política democrática no es equiparable simplemente a la movilización popular extensa y recurrente, o la participación focalizada. El consenso y los acuerdos son sólo un aspecto de los procesos políticos; en el reverso se encuentran los conflictos, los antagonismos y el desacuerdo. La democracia, entonces, tiene sentido en la medida en que se constituye como un régimen de gobierno que institucionaliza el procesamiento pacífico del desacuerdo político. Más aún: las libertades de asociación, ideas, comunicación y expresión están en la base de una vida pública participativa y abierta a la expresión legítima del desacuerdo. Las instituciones del régimen, entonces, deben servir como canales de expresión de puntos de vista distintos. Desde la perpectiva democrática, es necesario optimizar las condiciones que permiten la manifestación de opiniones contenciosas y los escenarios de resolución vinculante de los diferendos.

Para evaluar empíricamente estos elementos es habitual preguntarse si en un sistema dado existen elecciones regulares y un sistema de partidos competitivo que permita la rotación de las élites en los cargos públicos. Sin 
embargo, el principio de la contestabilidad política puede entenderse de una manera más amplia. Para extender el sentido de la contestabilidad democrática se le puede dar una interpretación deliberativa (Chambers, 2003). Desde este punto de vista, estará más apegada a los ideales democráticos una competencia política que, en primera instancia, ofrezca mejores condiciones de inclusión y equidad. Esto supone regular el financiamiento que obtienen los partidos políticos y candidatos, el acceso a los medios de comunicación y la conducción de las campañas electorales. En segundo lugar, la competencia política tendría que ser auténticamente deliberativa: debe ser un proceso de discusión que conduzca a identificar los fines de la política y a encontrar los medios idóneos para alcanzarlos. El espacio público se convierte en una arena de confrontación y disputa argumentales, regida por preceptos de justificación y responsabilidad, que está dirigida a formar e informar el juicio político colectivo que precede a la adopción de decisiones políticas y vinculantes.

En tercer lugar se ubica el control popular de la acción pública. Esta noción busca conectar los procesos de formación de la agenda pública con la toma de decisiones políticas, la implementación de las políticas públicas y la evaluación de sus resultados. La premisa es que si una democracia pretende ser un sistema de gobierno que sea congruente y responsivo con las preferencias de los ciudadanos, es necesario evaluar en qué medida y bajo qué circunstancias los resultados de la acción pública son congruentes con las preferencias mayoritarias de la ciudadanía. En este caso, la noción de acción pública alude a toda forma de acción estatal, gubernamental y social que esté dirigida a alcanzar metas colectivas, a través de leyes, estatutos, acuerdos, programas e instrumentos de coordinación concretos. El control popular, a su vez, es la conexión que se establece entre las preferencias de los ciudadanos y los procesos y los resultados políticos. Esta conexión, no obstante, está lejos de ser sencilla y directa. Por el contrario, depende de múltiples factores sociopolíticos e institucionales. Por ejemplo, depende de la estructura de configuración y agregación de intereses; esto es, de los sistemas de partidos. Depende también de las reglas que estructuran los procesos de toma de decisiones legislativas, del control de los representantes sobre la ejecución de las políticas públicas, y de la manera en que se evalúan los resultados de las políticas estatales y gubernamentales. En pocas palabras, el control 
popular abarca cuestiones relacionadas con los procesos de representación política, formación de mayorías gobernantes, rendición de cuentas y responsividad.

Para analizar el control democrático es necesario dar cuenta, en primer lugar, de la forma en que se construyen y representan los intereses y las preferencias de la ciudadanía. El concepto de representación política es complejo (Manin, 1997; Powell, 2004). Manin, Prezeworski y Stokes (1999) han mostrado que no existe un mecanismo eficaz para garantizar que las acciones de los representantes sean a la vez representativas y las que mejores promueven el interés público. El concepto de representación política, con todo, está estrechamente ligado al principio de inclusión democrática (Plotke, 1997; Urbinati y Warren, 2008), en la medida en que supone la existencia de procesos que "hacen presentes" las perspectivas, los intereses y las identidades políticas que hay en el espacio público en los espacios de decisión. En tal sentido, la representación política es un medio de control popular que se compone de elementos organizacionales, ideológicos e institucionales. Por un lado, para que exista representación popular se requiere que existan movimientos y organizaciones políticas que estructuren las ideologías y los programas que habrán de incorporar las demandas de la ciudadanía (Kitschelt y Wilkinson, 2007). Por el otro, la presencia y el peso relativo que tendrán los representantes y sus programas ideológicos en los procesos de decisión dependen de instituciones como las circunscripciones electorales, el tamaño de los distritos, las reglas de transformación de los votos en escaños, el tamaño de los parlamentos, etcétera (Gallagher y Mitchell, 2005; Nolhen, 2004).

Una segunda cuestión relacionada con el control popular de las acciones públicas es la que tiene que ver con las reglas y los procesos involucrados en las decisiones políticas, la formulación de las leyes y las políticas públicas. La cuestión está en cómo se constituyen, y sobre qué bases institucionales, las mayorías gobernantes. En los estudios sobre el presidencialismo, el parlamentarismo y las variantes intermedias (Linz, 1994; Shugart y Mainwaring, 1997; Cheibub, 2007), los modelos de representación democrática (Powell, 2000) y los modelos consensuales y mayoritarios de la democracia (Lijphart, 2000), queda de manifiesto que existen distintos arreglos institucionales que tienen ventajas y desventajas relativas. En gran medida, la discusión gira en torno a las consecuencias de dividir y 
fragmentar el poder, frente a los beneficios de concentrar la autoridad democrática. Sobre esta cuestión, Gerring y Thacker (2008) sostienen que los regímenes que cuentan con arreglos institucionales que contribuyen a lograr que la autoridad política sea convergente con una estructura centrípeta de decisión e implementación, al mismo tiempo que son representativos e inclusivos, son regímenes de los que se puede esperar mejores resultados en las políticas públicas. El modelo de la democracia consensual de Lijphart (2000) tiene un planteamiento semejante; sin embargo, Lijphart no esclarece el mecanismo que permite a la multiplicidad de actores y ramas institucionales cooperar y coordinarse políticamente. La tesis de Gerring y Thacker (2008) es que esto se consigue abriendo el espacio de decisión a múltiples fuerzas políticas, pero evitando fragmentar la autoridad pública. La tarea que queda abierta es la de indagar las cualidades de los mecanismos asociados a la constitución de las mayorías que deciden y gobiernan en representación del demos.

Dos cuestiones más que son necesarias para evaluar el control popular de la acción pública son los procesos de rendición de cuentas y la evaluación pública del desempeño de la democracia. La rendición de cuentas pública es un proceso político semánticamente denso, pues está asociado con varios ideales políticos y requiere múltiples sistemas institucionales para funcionar (Bovens, 2010). Entre otros elementos, implica que los representantes populares deben contar con instrumentos para vigilar que las agencias gubernamentales se conduzcan con apego a estándares de eficiencia, eficacia, legalidad y transparencia en la implementación de las leyes y las políticas públicas. Para valorar la calidad de una democracia es necesario tomar en cuenta esta dimensión: el control efectivo que tienen los representantes democráticos sobre la burocracia y las dependencias responsables de alcanzar los fines de la política pública. Asimismo, para evaluar el control popular sobre la acción pública es necesario remitirse a la evaluación que la propia ciudadanía realiza de las acciones estatales, gubernamentales o públicas. Los instrumentos requeridos para llevar a cabo esta tarea pueden ser diversos: sondeos de opinión, grupos focales, encuestas deliberativas, entre otros. La clave está en tomar el pulso de manera certera a la opinión pública. No se trata simplemente de medir la popularidad de los gobernantes, sino de sistematizar la manera en que los principales, los ciudadanos de a pie, valoran y juzgan el funcionamiento del gobierno que actúa en su nombre. 


\section{Consideraciones analíticas y agendas abiertas}

A lo largo de este trabajo hemos señalado que para evaluar la calidad de la democracia es necesario identificar los componentes que le dan vitalidad democrática a un régimen político. El argumento es que una democracia vital y robusta se manifiesta cuando las leyes, las políticas públicas y las acciones del gobierno se adoptan en condiciones en las que la participación política, la contestabilidad y la competencia son vigorosas, y cuando existen organizaciones, instituciones y procesos políticos que permiten a la ciudadanía y a sus representantes ejercer control sobre las decisiones y acciones públicas. Este planteamiento tiene diversas consecuencias analíticas.

En primer lugar, el análisis de la "calidad" de la democracia comienza a partir de una doble distinción: primeramente, es necesario diferenciar a los regímenes democráticos de los que no lo son - y de los que no lo son plenamente- En segundo lugar, es preciso distinguir a la democracia de otros conceptos, como el Estado de derecho, la burocracia o el buen gobierno. De esta manera, el análisis deberá enfocarse en los atributos del régimen que establecen diferencias en las cualidades de la política democrática. En este trabajo hemos señalado que esos atributos son los de participación, competencia y control popular de las acciones públicas, pero la discusión está abierta. El criterio a seguir es éste: la calidad de la democracia debe evaluarse en relación con lo que hace democrático al régimen.

Una vez identificados los componentes de la calidad de la democracia, lo habitual es recoger indicadores de distinta índole y arribar a una medida compuesta. Esto permite ordenar a los sistemas políticos en función de la puntuación que hayan alcanzado en un índice. Como ya se mencionó en la primera sección de este trabajo, una dificultad con este procedimiento es que asume que la calidad de la democracia es unidimensional. La discusión elaborada en este texto hace una apuesta distinta: para evaluar certeramente la calidad de la democracia es preciso reconocer que la democracia está compuesta por atributos que no convergen en torno a una misma dimensión. Por lo tanto, es necesario analizar cada uno de los componentes de la vitalidad democrática del régimen, sin asumir que es indispensable arribar a una medida sintética. Éste no es un llamado a dejar de usar medidas numéricas agregadas; antes bien, es un llamado a que los 
índices compuestos sean congruentes con la estructura de los conceptos que se están observando.

Por otra parte, si de lo que se trata es de distinguir cualidades, es preciso, todavía, dar un paso adelante: se puede convertir a cada uno de los componentes de la democracia en una arena de investigación en sí misma. Previsiblemente, un proceso de reconstrucción normativa de los conceptos de participación, competencia, representación y gobernanza democrática abran la puerta a múltiples términos y categorías. La ruta analítica que se está dibujando es ésta: la calidad de la democracia es una noción que invita a realizar estudios que utilicen descripciones densas y contextualizadas. Por ende, para dar cuenta de la calidad de un régimen en particular se requiere ofrecer un análisis robusto de la manera en que en ese régimen se manifiesta la participación, los procesos de contienda y oposición, la representación política y demás. Sería pertinente seleccionar por separado cada uno de esos componentes para desagregarlo conceptualmente y observarlo con detenimiento en estudios empíricos concretos.

Parecería que lo que se está sugiriendo es la adopción de una línea de investigación dirigida a producir estudios monográficos y descriptivos. Tal vez sea necesario trazar mejor la diferencia. Una tarea clave para el desarrollo de los estudios en torno a la calidad de la democracia es realizar análisis que sean producto de inmersiones profundas por parte de los analistas, pero que no pierdan de vista la centralidad que tiene en esta agenda la clarificación de los elementos normativos y la sistematización conceptual. Ésta es una pieza clave para proseguir con el desafío mayor: formular explicaciones teóricas acerca de las manifestaciones y los grados variables de la calidad de la democracia.

La sistematización conceptual debe desembocar en la formulación de explicaciones causales de la calidad de la democracia. De manera congruente con lo que se ha venido discutiendo, si lo que se teoriza son los componentes concretos de la vitalidad democrática se puede alcanzar mayor capacidad explicativa. Es más plausible que se puedan formular teorías de alcance medio acerca de algunos de los componentes de la democracia, a que se pueda desarrollar una teoría amplia que abarque a todos los componentes de la calidad democrática de manera simultánea.

Existen tres agendas de investigación concreta que cabría explorar: la primera implica que los atributos de la calidad de la democracia se 
convierten en variables dependientes. Por ejemplo, se pueden estudiar los impactos que tienen el Estado de derecho, la burocracia, las dinámicas asociativas y el desempeño económico del gobierno, entre otros factores, en los componentes concretos de la calidad democrática. Una segunda ruta es asumir que los componentes de la calidad democrática son variables independientes. Esto conduciría a evaluar el efecto que tienen los componentes de la vitalidad democrática en otras variables de interés: la legitimidad de la democracia, la satisfacción con el desempeño del régimen, la pertinencia y eficacia de las políticas públicas, el desarrollo humano, el crecimiento económico, entre otras. En tercer lugar, sería pertinente explorar las relaciones empíricas entre los distintos componentes de la calidad democrática: ¿cómo se asocian, en el marco de regímenes poliárquicos, los atributos de la participación y la contestabilidad políticas? ¿Qué conexiones existen entre los componentes de la representación política y los procesos de autorización y formulación de políticas públicas?

Para concluir, una reflexión adicional: el estudio de las cualidades y la vitalidad de la democracia no puede desligarse de la teoría política normativa. La densidad semántica y la inserción de los conceptos en tradiciones concretas de pensamiento son elementos que pueden contribuir al análisis empírico. En gran medida, uno de los desafíos más importantes para el estudio de la calidad de la democracia es mantener un diálogo productivo y sistemático entre la teoría política normativa, el desarrollo de conceptos y la investigación política empírica.

\section{Bibliografía}

Austen-Smith, David y Jeffrey Banks (2000), Positive Political Theory I: Collective Preference, Michigan University Press, Ann Arbor.

Bergman, Marcelo y Lawrence Whitehead (2009), Criminalty, Public Security and the Challenges to Democracy in Latin America, Hellen Kellogg Institute for International Studies, University of Notre Dame Press. Notre Dame.

Bovens, Mark (2010), "Two Concepts of Accountability: Accountability as a Virtue and as a Mechanism”, West European Politics, vol. 33, núm. 5, pp. 946-967.

Chambers, Simone (2003), "Deliberate Democratic Theory", Annual Review of Political Science, vol. 5, pp. 307-326. 
Cheibub, José Antonio (2007), Presidentialism, Parliamentarism, and Democracy, Cambridge University Press, Cambridge.

Christiano, Thomas (2004), "The Authority of Democracy", Journal of Political Philosophy, vol. 12, núm. 3, pp. 266-290.

— (1996), The Rule of the Many: Fundamental Issues in Democratic Theory, Westview Press, Boulder Colorado, Estados Unidos.

Collier, David y Steven Levitsky (1997), "Democracies with Adjectives: Conceptual Innovation in Comparative Research", World Politics, vol. 49, núm. 3, pp. 430451.

Collier, David, Fernando Daniel Hidalgo y Andra Olivia Maciuceanu (2006), Journal of Political Ideologies, vol. 11, núm. 3, pp. 211-246.

Coppedge, Michael (2007), "Thickening thin Concepts: Issues in Large-N Data Generation", en G. Munck (ed.), Regimes and Democracy in Latin America: Theories and Methods, Oxford University Press, Oxford.

Croissant, Aurel y Wolfang Merkel (2004), "Introduction. Democratization in the Early Twentieth-First Century”, Democratization, vol. 11, núm. 5, pp. 1-9.

Dahl, Robert (1989), Democracy and its Critics, Yale University Press, New Heaven. — (1971), Poliarchy: Participation and Opposition, Yale University Press, New Heaven. Diamond, Larry (1999), Developing Democracy: Towards Consolidation, John Hopkins University Press, Baltimore.

Dryzek, John, Bonnie Honig y Anne Phillips (2006), “Introduction”, en J. Dryzek,

B. Honig y A. Phillips (coords.), Oxford Handbook of Political Theory, Oxford University Press, Oxford.

Ferejohn John y Pasquale Pasquino (2003), "Rule of Democracy and Rule of Law", en J. M. Maravall y A. Przeworski (eds.), Democracy and The Rule of Law, Cambridge University Press, Cambridge.

Fung, Archon (2006), "Varieties of Participation in Complex Governance", Public Administration Review, núm. 66, pp. 66-75.

Gallagher, Michael y Paul Mitchell (eds.), (2005). The Politics of Electoral Systems, Oxford University Press, Oxford.

Gerring, John y Strom Thacker (2008), A Centripetal Theory of Democratic Governance, Cambridge University Press, Cambridge.

Habermas, Jürgen (1998), Facticidad y validez, Trotta, Madrid.

Held, David (2006), Models of Democracy, Stanford University Press, Stanford.

IDD-Lat (2012), Índice de Desarrollo Democrático de América Latina, Fundación Konrad-Adenauer, PoliLat, en <http://www.idd-lat.org/>. Fecha de consulta: 18 de marzo de 2013.

Kitschelt, Herbert y Steven Wilkinson (2007), "Citizen-Politician Linkages: An Introduction", en Herbert Kitschelt y Steven Wilkinson (eds.), Patrons, Clientes, and Policies: Patterns of Democratic Accountability and Political Competition, Cambridge University Press, Cambridge. 
Landmann, Todd (2009), Evaluar la calidad de la democracia: una introducción al marco de IDEA Internacional, (en colaboración con David Betham, Edzia Carvalho y Stuart Weir), Instituto Internacional para la Democracia y la Asistencia Electoral, Estocolmo, Suecia.

Levine, Daniel y José Enrique Molina (2007), "La calidad de la democracia en América Latina: una visión comparada”, América Latina Hoy, núm. 45, pp. 17-46.

Levitsky, Steven y Lucan A. Way (2010), Competitive Authoritarianism: Hybrid Regimes After the Cold War, Cambridge University Press, Cambridge.

Lijphart, Arend (2000), Modelos de democracia, Ariel, Barcelona.

Linz, Juan (1994), "Presidential or Parliamentary Democracy: Does it Makes a Difference?", en Juan J. Linz y Arturo Valenzuela (eds.), The Failure of Presidential Democracy, The John Hopkins University Press, Baltimore.

Linz, Juan y Alfred Stepan (1996), Problems of Democratic Transition and Consolidation, The John Hopkins University Press, Baltimore.

Mainwaring, Scott y Christopher Welna (eds.) (2003), Democratic Accountability in Latin America, Oxford University Press, Oxford.

Mainwaring, Scott, Timothy Scully y Jorge Vargas-Cullel (2010), "Measuring Success in Democratic Governance", en Scott Mainwaring y Timothy Scully (eds.), Democratic Governance in Latin America, Stanford University Press, Stanford.

Mainwaring, Scott, Daniel Brinks y Aníbal Pérez-Liñán (2007), "Classifying Political Regimes in Latin America, 1945-2004”, en G. Munck (ed.), Regimes and Democracy in Latin America: Theories and Methods, Oxford University Press, Oxford.

Mainwaring, Scott y Aníbal Pérez-Liñan (2008), “Regimen Legacies and Democratization: Explaining Variance in the Level of Democracy in Latin America, 1978-2004", documento de trabajo, núm. 354, The Helen Kellog Institute for International Studies.

Manin, Bernard (1997), The Principles of Representative Government, Cambridge University Press, Cambridge.

—. Adam Prezeworski y Susan Stokes (1999), "Introduction”, en Adam Przeworski, Susan Stokes y Bernard Manin (eds.), Democracy, Accountability and Representation, Cambridge University Press, Cambridge.

Maravall, José María y Adam Przeworski (eds.) (2003), Democracy and the Rule of Law, Cambridge University Press, Cambridge.

Mazzuca, Sebastian L. (2007), "Reconceptualizing Democratization: Access to Power Versus Excercise of Power", en G. Munck (ed.), Regimes and Democracy in Latin America: Theories and Methods, Oxford University Press, Oxford.

Méndez, Juan E., Paulo Sergio Pinheiro y Guillermo O'Donnell (1999). The (Un) rule of Law $\Xi$ The Underpriviliged in Latin America, Hellen Kellogg Institute for International Studies, University of Notre Dame Press, Notre Dame. 
Morlino, Leonardo (2005), Democracias y democratizaciones, CEPCOM, México.

— (2011), Changes for Democracy: Actors, Structures, Process, Oxford University Press, Oxford.

Munck, Gerardo (2004), "Democratic Politics in Latin America: New Debates and Research Frontiers”, Annual Review of Political Science, núm. 7, pp. 437-462.

(2006), "Drawing Boundaries: How to Craft Intermediate Regime Categories”, en A. Schedler (ed.), Electoral Authoritarianism: The Dynamics of Unfree Competition, Lynne Reiner, Boulder Colorado.

— (2007), "The Study of Politics and Democracy: Touchstones of a Research Agenda”, en G. Munck (ed.), Regimes and Democracy in Latin America: Theories and Methods, Oxford University Press, Oxford.

— (2011), "Los estándares de la democracia: hacia una formulación de la cuestión democrática en América Latina”, Journal of Democracy en Español, vol. 3, pp. 22-41.

Munck, Gerardo y Jay Verkuilen (2002), "Conceptualizing \& Measuring Democracy: Evaluation Alternative Indices”, Comparative Political Studies, vol. 35, núm. 1, pp. 5-34.

Nolhen, Dieter (2004), Sistemas electorales y partidos políticos, Fondo de Cultura Económica, México.

O’Donnell, Guillermo (2004), "Human Development, Human Rights, and Democracy”, en Guillermo O’Donnell, Jorge Vargas-Cullel y Osvaldo Iazzetta (eds.), The Quality of Democracy: Theory and Applications, Hellen Kellogg Institute for International Studies, University of Notre Dame Press, Notre Dame.

O’Donnell, Guillermo, Jorge Vargas-Cullel y Osvaldo Iazzetta (eds.) (2004), The Quality of Democracy: Theory and Applications, University of Notre Dame Press, Notre Dame.

Plotke, David (1997), "Representation is Democracy", Constellations, núm. 4, pp. 19-34.

PNUD (2004), La democracia en América Latina: hacia una democracia de ciudadanas y ciudadanos, PNud, Buenos Aires.

— (2010), Nuestra democracia, PNud, Organización de los Estados Americanos, Fondo de Cultura Económica, México.

Powell, Bingham G. Jr. (2004), "Political Representation in Comparative Politics", Annual Review of Political Science, núm. 7, pp. 273-96.

- (2000), Elections as Instruments of Democracy: Mayoritarian and Proportional Visions, Yale University Press, Yale.

Przeworski, Adam (1991), Democracy and the Market: Political and Economic Reforms in Eastern Europe and Latin America, Cambridge University Press, Cambridge.

— (2010), Democracy $\Xi^{2}$ the Limits of Self-Government, Cambridge University Press, Cambridge.

Rawls, John (1999), A Theory of Justice, Belknap Harvard University Press, Harvard. 
Riker, William (1982), Liberalism Against Populism, W. H. Freeman, San Francisco. Sartori, Giovanni (1970), "Concept Misformation in Comparative Politics", The American Political Science Review, vol. 64, núm. 4, pp. 1033-1053.

Schedler, Andreas (2006), "The Logic of Electoral Authoritarianism", en A. Schedler (ed.), Electoral Authoritarianism: The Dynamics of Unfree Competition, Lynne Reiner, Boulder Colorado.

Schumpeter, Joseph (1971), Capitalism, Socialism and Democracy, Harper Perennial, Nueva York [1950, 3a. edición].

Shugart, Matthew S. y Scott Mainwaring (eds.) (1997), Presidentialism and Democracy in Latin America, Cambridge University Press, Cambridge.

Smith, Graham (2009), Democratic Innovations: Designing Institutions for Citizen Participation, Cambridge University Press, Cambridge.

Urbinati, Nadia y Mark Warren (2008), "The Concept of Representation in Contemporary Democratic Theory", Annual Review of Political Science, vol. 11, pp. 387-412.

Warren, Mark (1996), "What Should we Expect from More Democracy? Radically Democratic Responses to Politics", Political Theory, vol. 24, núm 2, pp. 241270.

(2006), "Democracy and the State", en John Dryzek, Bonnie Honig y Anne Phillips (eds.), The Oxford Handbook of Political Theory, Oxford University Press, Oxford, 2006.

Young, Iris Marion (2000), Inclusion and Democracy, Oxford University Press, Oxford.

Zakaria, Fareed (2003), The Future of Freedom: Illiberal Democracy at Home छ Abroad, W.W. Norton \& Company, Nueva York.

Artículo recibido el 12 de julio de 2012. Segunda versión aprobada el 10 de junio de 2013. 